\title{
Performance Analysis of Amplify-and-Forward Relaying Aided Cooperative Spatial Modulation over Rayleigh Channel
}

\author{
Qing Pan \\ College of Electronic and \\ Information Engineering \\ Nanjing University of Aeronautics \\ and Astronautics \\ Nanjing, China 211106 \\ yu_zhouli2009@126.com
}

\author{
Xiangbin $\mathrm{Yu}$ \\ College of Electronic and \\ Information Engineering \\ Nanjing University of Aeronautics \\ and Astronautics \\ Nanjing, China 211106 \\ yxb_xwy@hotmail.com
}

\author{
Yaping $\mathrm{Hu}$ \\ College of Electronic and \\ Information Engineering \\ Nanjing University of Aeronautics \\ and Astronautics \\ Nanjing, China 211106
}

\author{
Cheng Wang \\ College of Electronic and \\ Information Engineering \\ Nanjing University of Aeronautics \\ and Astronautics
}

\author{
Xi Wang \\ College of Electronic and \\ Information Engineering \\ Nanjing University of Aeronautics \\ and Astronautics
}

\begin{abstract}
By combing SM with the amplify-and-forward (AF) cooperative communication, the cooperative SM scheme with AF (AF-SM) is presented for obtaining superior performance. The bit error rate (BER) performance of AF-SM is investigated over Rayleigh channels. According to the performance analysis, a tightly upper bound of the output SNR is given, and the corresponding probability density function (PDF) is derived by means of the moment generating function (MGF). Based on the obtained PDF and MGF, the error probability of antenna index estimation $\left(P_{a}\right)$ and the error probability of symbol estimation $\left(P_{d}\right)$, which constitute the overall average $\mathrm{BER}$, are derived, respectively. As a result, tightly approximate closed-form expressions of the $P_{a}$ and $P_{d}$ are obtained, respectively. With these expressions, the closed-form BER is achieved. These theoretical expressions can match the simulations well, and thus the BER performance of AF-SM can be effectively evaluated. Simulation results verify the effectiveness of the theoretical analysis, and the BER performance can be improved by increasing the receive antennas.
\end{abstract}

\section{KEYWORDS}

Spatial modulation, cooperative communication, amplify and forward relaying, average BER

ACM Reference format:

Qing Pan, Xiangbin Yu, Yaping Hu, Cheng Wang, and Xi Wang. 2017. Performance Analysis of Amplify-and-Forward Relaying Aided Cooperative Spatial Modulation over Rayleigh Channel. In

Permission to make digital or hard copies of all or part of this work for personal or classroom use is granted without fee provided that copies are not made or distributed for profit or commercial advantage and that copies bear this notice and the full citation on the first page. To copy otherwise, to republish, to post on servers or to redistribute to lists, requires prior specific permission and/or a fee.

MOBIMEDIA 2017, July 13-14, Chongqing, People's Republic of China Copyright (@) 2017 EAI 978-1-63190-156-0
Proceedings of EAI International Conference, Chong Qing, China, July 2017 (MOBIMEDIA), 6 pages.

DOI: $10.475 / 123 \_4$

\section{INTRODUCTION}

With a high demand for wireless products, it becomes imperative for next generation wireless communication to provide high data rate and link reliability. Cooperative communication technology is proposed to improve the spectral efficiency and network coverage of the future wireless communication systems, and has earned much more attention [1]. Amplifyand-forward (AF) protocol [2], as one of effective cooperative protocols, has been widely used for cooperative communication. In this protocol, relay amplifies and transfers the signals sent by source, and then the receiver combines the signals sent by source and relay, and makes a final decision on the transmitted bit.

Spatial modulation (SM), employing active transmit antenna indices as added constellation points to convey information, can eliminate the need for transmit antenna synchronization and inter-channel interference, and has attracted much interests. SM scheme was firstly proposed by Mesle$\mathrm{h}$ et.al in [3]. In this scheme, only one antenna is active at any time slot and the transmitted bits are mapped to constellation symbols and active antenna index. At the receiver, the maximum likelihood (ML) detector is used to demodulate the signals and estimate the transmitted symbol and the transmit antenna index. However, the conventional SM scheme does not considering the superiority of cooperative communication, and thus its performance will be limited, especially for long-distance communication.

For this reason, considering the simplicity of SSK (a special SM, where only active antenna index conveys information), some relay-aided SSK schemes have been presented [4-6]. With these scheme, the spectrum efficiency can be improved. An AF relay aided space-time SSK was presented in [4] to mitigate the effect of deep fading. In [5, 6], dual-hop 
AF relaying aided SSK schemes were proposed, and the corresponding bit error rate (BER) performance was analyzed, and the upper-bounds of BER were derived. However, SSK only uses the transmit antenna index for carrying information, and does not consider the superiority of constellation modulation. Thus, the throughput of the cooperative SSK schemes remains somewhat limited.

Based on the analysis above, the relay-aided cooperative $\mathrm{SM}$ scheme is presented in this paper, where AF is used for cooperative protocol considering its effectiveness. We analyze the performance of this AF relay-aided SM (AF-SM), and give the output SNR at the destination and its tightly upper bound. According to this, using the moment generating function (MGF), the probability density function (PDF) of upper bound of the output SNR is derived. With the PDF and MGF, the closed-form expressions of the error probability of antenna index estimation and the error probability of symbol estimation, which constitute the overall average BER, are obtained. Thus, the tightly approximate closedform expression of average BER is achieved. Simulation results show that the derived theoretical expressions are valid, and agree well with the corresponding simulations.

The notations throughout this paper are as follows. Bold upper case and lower case letters denote matrices and column vectors, respectively. The superscripts $(\cdot)^{H}$ and $(\cdot)^{T}$ denote the Hermitian transposition and transposition, respectively.

\section{CHANNEL AND SYSTEM MODELS}

By combining spatial modulation and cooperative communication with AF protocol, a cooperative AF-SM system is presented. The system model is illustrated in Fig.1, and it consists of one source with $N_{t}$ antennas, one destination with $N_{r}$ antennas, and one relay node with single antenna. It is assumed that there is a direct link between the source and the destination, and all the nodes are half-duplex transmission.

As shown in Fig.1, at the source, the SM mapper assigns $m=\log _{2}\left(M N_{t}\right)$ bits for data transmission, where $\log _{2} N_{t}$ bits determine the transmit antenna index $j, j \in\left[1: N_{t}\right]$, and $\log _{2} M$ bits is used for $M$-ary constellation mapping. The output of SM mapper can be expressed as [7]

$$
\mathbf{x}_{j q}=\left[\begin{array}{llllll}
0 & 0 & \cdots & x_{q} & \cdots & 0
\end{array}\right]^{T}
$$

where $\mathbf{x}_{j q}$ is the $N_{t}$ dimensional symbol vector, $x_{q}$ is in the $j$ th column of $\mathbf{x}_{j q}$ and denotes the $q$ th symbol from an $M$ ary constellation with $q \in[1: M]$. The system transmission is divided into phases. In phase 1 , the source transmits $x_{q}$ via the $j$ th antenna to the relay and the destination. The received signal at the relay and the destination can be, respectively, expressed as

$$
\begin{gathered}
y_{s r}=\sqrt{P_{s}} \mathbf{h}_{s r}^{T} \mathbf{x}_{j q}+n_{s r} \\
\mathbf{y}_{s d}=\sqrt{P_{s}} \mathbf{H}_{s d} \mathbf{x}_{j q}+\mathbf{n}_{s d}
\end{gathered}
$$

where $P_{s}$ is the transmitted power of the source, $\mathbf{h}_{s r}$ is channel vector of the source-to-relay link, and $\mathbf{H}_{s d}$ is channel matrix of the source-to-destination link.

In phase 2, the relay amplifies and transfers the signal received in phase 1 . The received signal of the $i$ th receive antenna at the destination can be given by

$$
y_{r d_{i}}=h_{r d_{i}}\left(A y_{s r}\right)+n_{r d_{i}}, i \in\left[1: N_{r}\right]
$$

where $h_{r d_{i}}$ is the channel coefficient of the relay to the $i$ th receive antenna link. The entries of $\mathbf{h}_{s r}, \mathbf{H}_{s d}$ and $h_{r d_{i}}$ are independent and identically distributed (i.i.d.) complex Gaussian random variables with zero mean and unit variance. The noise $n_{s r}, \mathbf{n}_{s d}$ and $n_{r d_{i}}$ are additive white Gaussian noise (AWGN) with a variance of $N_{0} . A$ is the amplification factor and can be written as

$$
A=\sqrt{P_{r} /\left(P_{s}\left|h_{s r}^{j}\right|^{2}+N_{0}\right)}
$$

where $P_{r}$ is the output power of the relay. After normalization processing, the equation (4) becomes

$$
y_{r d_{i}}=\sqrt{G_{i}} \mathbf{h}_{s r}^{T} \mathbf{x}_{j q}+n_{i}
$$

where $G_{i}=P_{s} A^{2}\left|h_{r d_{i}}\right|^{2} /\left(A^{2}\left|h_{r d_{i}}\right|^{2}+1\right)$, and $n_{i}=\left(A h_{r d_{i}} n_{s r}+\right.$ $\left.n_{r d_{i}}\right) / \sqrt{A^{2}\left|h_{r d_{i}}\right|^{2}+1}$.

With the obtained received signals, the optimal SM detector based on the ML principle is given by

$$
\begin{aligned}
{\left[\hat{j}, x_{\hat{q}}\right] } & =\underset{j, q}{\operatorname{argmin}}\left[P_{s}\left\|\mathbf{h}_{s d}^{j} x_{q}\right\|_{F}^{2}-2 \sqrt{P_{s}} \operatorname{Re}\left(\mathbf{y}_{s d}^{H} \mathbf{h}_{s d}^{j} x_{q}\right)\right. \\
& \left.+\sum_{i=1}^{N_{r}} G_{i}\left|h_{s r}^{j} x_{q}\right|^{2}-2 \sqrt{G_{i}} \operatorname{Re}\left(y_{r d_{i}}^{*} h_{s r}^{j} x_{q}\right)\right]
\end{aligned}
$$

where $\mathbf{h}_{s d}^{j}$ and $h_{s r}^{j}$ denotes the $j$ th column of $\mathbf{H}_{s d}$ and $j$ th element of $\mathbf{h}_{s r}^{T}, \hat{j}$ and $x_{\hat{q}}$ represent the estimated transmit antenna index and symbol, respectively. With this optimal detector, the transmit antenna index and the transmitted symbol can be jointly detected well.

The effective output SNR at the destination can be expressed as [8]

$$
\gamma_{o u t}=\gamma_{S D}+\frac{\gamma_{S R} \gamma_{R D}}{1+\gamma_{S R}+\gamma_{R D}}
$$

where $\gamma_{S D}, \gamma_{S R}$ and $\gamma_{R D}$ are the instantaneous SNRs of the source-to-destination link, the source-to-relay link and the relay-to-destination link, respectively.

Considering the Rayleigh channel, the PDF of $\gamma_{S D}$ is written as

$$
f_{\gamma_{S D}}(\gamma)=\frac{1}{\Gamma\left(N_{r}\right) \bar{\gamma}_{S D}}\left(\frac{\gamma}{\bar{\gamma}_{S D}}\right)^{N_{r}-1} \exp \left(-\frac{\gamma}{\bar{\gamma}_{S D}}\right)
$$

and the cumulative distribution function $(\mathrm{CDF})$ of $\gamma_{S R}$ and $\gamma_{R D}$ are, respectively, given by

$$
\begin{gathered}
F_{\gamma_{S R}}(\gamma)=1-\exp \left(-\frac{\gamma}{\bar{\gamma}_{S R}}\right) \\
F_{\gamma_{R D}}(\gamma)=1-\exp \left(-\frac{\gamma}{\bar{\gamma}_{R D}}\right) \sum_{k=0}^{N_{r}-1} \frac{1}{k !}\left(\frac{\gamma}{\bar{\gamma}_{R D}}\right)^{k}
\end{gathered}
$$

For convenient analysis, a tightly upper bound of the output SNR is employed, and shown as [9]

$$
\gamma_{\text {out }} \leq \gamma_{S D}+\min \left(\gamma_{S D}, \gamma_{R D}\right)=\gamma_{S D}+\gamma_{S R D}=\gamma_{u p}
$$

This upper-bound is shown to be quite accurate for $\gamma_{\text {out }}[8,9]$, and it facilitates the derivations of the PDF, CDF and MGF, which will be used for the following performance analysis. 


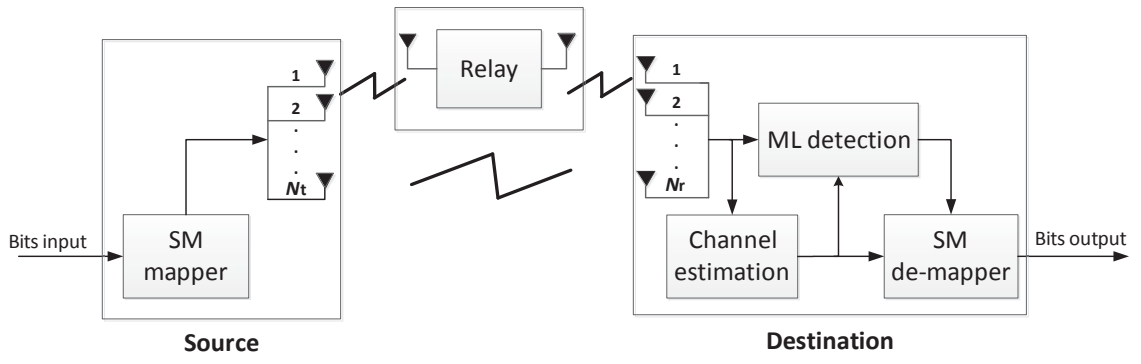

Figure 1: AF-SM system model

Considering that $\gamma_{S D}$ and $\gamma_{S R D}$ are independent, the MGF of $\gamma_{u p}$ is equal to the product of the MGF of $\gamma_{S D}$ and the MGF of $\gamma_{S R D}$. Thus, it can be expressed as

$$
M_{\gamma_{u p}}(s)=M_{\gamma_{S D}}(s) M_{\gamma_{S R D}}(s)
$$

Based on (9), the MGF of $\gamma_{S D}$ is written as

$$
M_{\gamma_{S D}}(s)=\int_{0}^{\infty} e^{-s \gamma} f_{\gamma_{S D}}(\gamma) d \gamma=\left(1+\bar{\gamma}_{S D} s\right)^{-N_{r}}
$$

In order to obtain the MGF of $\gamma_{S R D}$, we will derive the CDF and the PDF of $\gamma_{S R D}$ firstly. Assuming that $\gamma_{S R}$ and $\gamma_{R D}$ are independent, the CDF of $\gamma_{S R D}=\min \left(\gamma_{S R}, \gamma_{R D}\right)$ can be expressed as [10]

$$
F_{\gamma_{S R D}}(\gamma)=1-\exp \left(-\frac{\gamma}{\bar{\gamma}_{C}}\right) \sum_{n=0}^{N_{r}-1} \frac{1}{n !}\left(\frac{\gamma}{\bar{\gamma}_{R D}}\right)^{n}
$$

where $\bar{\gamma}_{C}=\frac{\bar{\gamma}_{S R} \bar{\gamma}_{R D}}{\bar{\gamma}_{S R}+\bar{\gamma}_{R D}}$. Then, with (15), taking the derivative of $F_{\gamma_{S R D}}(\gamma)$ with respect to $\gamma$ yields

$$
\begin{aligned}
& f_{\gamma_{S R D}}(\gamma)=\frac{1}{\Gamma\left(N_{r}\right) \bar{\gamma}_{C}}\left(\frac{\gamma}{\bar{\gamma}_{R D}}\right)^{N_{r}-1} \exp \left(-\frac{\gamma}{\bar{\gamma}_{C}}\right) \\
& +\left(\frac{1}{\bar{\gamma}_{C}}-\frac{1}{\bar{\gamma}_{R D}}\right) \exp \left(-\frac{\gamma}{\bar{\gamma}_{C}}\right) \sum_{n=0}^{N_{r}-2} \frac{1}{n !}\left(\frac{\gamma}{\bar{\gamma}_{R D}}\right)^{n}
\end{aligned}
$$

Equation (16) is the PDF of $\gamma_{S R D}$. By setting $N_{r}=1$ to (16), we can obtain the PDF of $\gamma_{S R D}$ for single receive antenna.

Using (16), the MGF of $\gamma_{S R D}$ can be obtained as

$$
\begin{gathered}
M_{\gamma_{S R D}}(s)=\frac{1}{\bar{\gamma}_{C} \bar{\gamma}_{R D}{ }^{N_{r}-1}}\left(s+\frac{1}{\bar{\gamma}_{C}}\right)^{-N_{r}} \\
+\left(\frac{1}{\bar{\gamma}_{C}}-\frac{1}{\bar{\gamma}_{R D}}\right) \sum_{n=0}^{N_{r}-2} \frac{1}{\bar{\gamma}_{R D}{ }^{n}}\left(s+\frac{1}{\bar{\gamma}_{C}}\right)^{-(n+1)}
\end{gathered}
$$

Substituting (17) and (14) into (13), the MGF of $\gamma_{u p}$ can be expressed as

$$
\begin{aligned}
M_{\gamma_{u p}}(s) & =\frac{1}{\bar{\gamma}_{C} \bar{\gamma}_{R D}^{N_{r}-1} \bar{\gamma}_{S D} N_{r}}\left(s+\frac{1}{\bar{\gamma}_{C}}\right)^{-N_{r}}\left(s+\frac{1}{\bar{\gamma}_{S D}}\right)^{-N_{r}} \\
& +\frac{1}{\bar{\gamma}_{S D}{ }^{N_{r}}}\left(\frac{1}{\bar{\gamma}_{C}}-\frac{1}{\bar{\gamma}_{R D}}\right) \\
& \cdot \sum_{n=0}^{N_{r}-2} \frac{1}{\bar{\gamma}_{R D}^{n}}\left(s+\frac{1}{\bar{\gamma}_{C}}\right)^{-(n+1)}\left(s+\frac{1}{\bar{\gamma}_{S D}}\right)^{-N_{r}}
\end{aligned}
$$

With (18), using the inverse Laplace transform, the PDF of $\gamma_{u p}$ can be obtained as

$$
\begin{aligned}
& f_{\gamma_{u p}}(\gamma)=\int_{0}^{\infty} e^{s \gamma} M_{\gamma_{u p}}(s) d s=\frac{1}{\bar{\gamma}_{C} \bar{\gamma}_{R D}{ }^{N_{r}-1} \bar{\gamma}_{S D} N_{r}} . \\
& {\left[\sum_{i=0}^{N_{r}-1} \frac{E_{N_{r}-i} \gamma^{N_{r}-i-1}}{\left(N_{r}-i-1\right) !} e^{-\frac{\gamma}{\bar{\gamma}_{C}}}+\sum_{j=0}^{N_{r}-1} \frac{B_{N_{r}-j} \gamma^{N_{r}-j-1}}{\left(N_{r}-j-1\right) !} e^{-\frac{\gamma}{\bar{\gamma}_{S D}}}\right]} \\
& +\frac{1}{\bar{\gamma}_{S D} N_{r}}\left(\frac{1}{\bar{\gamma}_{C}}-\frac{1}{\bar{\gamma}_{R D}}\right) \sum_{n=0}^{N_{r}-2} \frac{1}{\bar{\gamma}_{R D}^{n}}\left[\sum_{p=0}^{n} \frac{C_{n+1-p} \gamma^{n-p}}{(n-p) !} e^{-\frac{\gamma}{\bar{\gamma}_{C}}}\right. \\
& \left.+\sum_{q=0}^{N_{r}-1} \frac{D_{N_{r}-q} \gamma^{N_{r}-q-1}}{\left(N_{r}-q-1\right) !} e^{-\frac{\gamma}{\bar{\gamma}_{S D}}}\right]
\end{aligned}
$$

where $E_{N_{r}-i}=\left.\frac{1}{i !}\left[\frac{1}{\left(s+1 / \bar{\gamma}_{S D}\right)^{N_{r}}}\right]^{(i)}\right|_{s=-1 / \bar{\gamma}_{C}}$,

$$
\begin{aligned}
& B_{N_{r}-j}=\left.\frac{1}{j !}\left[\frac{1}{\left(s+1 / \bar{\gamma}_{C}\right)^{N_{r}}}\right]^{(j)}\right|_{s=-1 / \bar{\gamma}_{S D},}, \\
& C_{n+1-p}=\left.\frac{1}{p !}\left[\frac{1}{\left(s+1 / \bar{\gamma}_{S D}\right)^{N_{r}}}\right]^{(p)}\right|_{s=-1 / \bar{\gamma}_{C}}, \\
& D_{N_{r}-q}=\left.\frac{1}{q !}\left[\frac{1}{\left(s+1 / \bar{\gamma}_{C}\right)^{n+1}}\right]^{(q)}\right|_{s=-1 / \bar{\gamma}_{S D}} .
\end{aligned}
$$

Using this PDF, we will provide the performance analysis in next section.

\section{PERFORMANCE ANALYSIS}

In this section, we will give the performance analysis of cooperative AF-SM in Rayleigh fading channel, and derive an approximate BER expression. At the destination, the SM detector estimates the active transmit antenna index and transmitted symbol. As a result, the SM performance depends on the error rates of these two detection processes. Let $P_{a}$ be the error probability of transmit antenna index estimation given that the symbol is perfectly detected and $P_{d}$ be the error probability of symbol estimation given that the transmit antenna index is perfectly detected, then the overall average BER of the system can be tightly approximated as

$$
P_{e} \approx 1-\left(1-P_{a}\right)\left(1-P_{d}\right)
$$

In what follows, we will derive the $P_{a}$ and $P_{d}$, respectively. Assuming the transmitted symbol is perfectly detected, $P_{a}$ is given as [3]

$$
P_{a} \leq \sum_{l=1}^{N_{t}} \sum_{q=1}^{M} \sum_{\tilde{l}=1}^{N_{t}} \frac{N(l, \widetilde{l})}{M N_{t} \log _{2}\left(N_{t}\right)} P \operatorname{EP}\left(x_{l} \rightarrow x_{\tilde{l}}\right)
$$




$$
\begin{gathered}
P_{d, M Q A M}=\sum_{l}^{\pi(M)} \frac{\alpha_{l}}{\bar{\gamma}_{C} \bar{\gamma}_{R D}^{N_{r}-1} \bar{\gamma}_{S D}^{N_{r}}}\left\{\sum_{i=0}^{N_{r}-1} E_{N_{r}-i} \bar{\gamma}_{C}^{N_{r}-i}\left[1-\sqrt{\beta_{l} / \pi} \sum_{p=0}^{N_{r}-i-1} \frac{\Gamma(p+1 / 2) w_{C}-(p+1 / 2)}{\bar{\gamma}_{C}^{p} \cdot p !}\right]+\sum_{j=0}^{N_{r}-1} B_{N_{r}-j} \bar{\gamma}_{S D}^{N_{r}-j}\right. \\
\cdot\left[1-\sqrt{\beta_{l} / \pi} \sum_{q=0}^{N_{r}-j-1} \frac{\Gamma(q+1 / 2) w_{S D}-(q+1 / 2)}{\bar{\gamma}_{S D}{ }^{q} \cdot q !}\right]+\sum_{l}^{\pi(M)} \frac{\alpha_{l}}{\bar{\gamma}_{S D}^{N_{r}}}\left(\frac{1}{\bar{\gamma}_{C}}-\frac{1}{\bar{\gamma}_{R D}}\right) \sum_{n=0}^{N_{r}-2} \frac{1}{\bar{\gamma}_{R D}{ }^{n}}\left\{\sum_{x=0}^{n} C_{n+1-x} \bar{\gamma}_{C}^{n+1-x}\right. \\
\left.\cdot\left[1-\sqrt{\beta_{l} / \pi} \sum_{a=0}^{n-x} \frac{\Gamma(a+1 / 2) w_{C}-(a+1 / 2)}{\bar{\gamma}_{C}{ }^{a} \cdot a !}\right]+\sum_{y=0}^{N_{r}-1} D_{N_{r}-y} \bar{\gamma}_{S D}^{N_{r}-y}\left[1-\sqrt{\beta_{l} / \pi} \sum_{b=0}^{N_{r}-y-1} \frac{\Gamma(b+1 / 2) w_{S D}-(b+1 / 2)}{\bar{\gamma}_{S D}{ }^{b} \cdot b !}\right]\right\}
\end{gathered}
$$

where $N(l, \widetilde{l})$ is the number of bits in error between transmit antenna index $l$ and the estimated transmit antenna index $\widetilde{l}$ and $\operatorname{PEP}\left(x_{l} \rightarrow x_{\widetilde{l}}\right)$ denotes the pairwise error probability (PEP), which can be expressed as

$$
\begin{aligned}
& \operatorname{PEP}\left(x_{l} \rightarrow x_{\tilde{l}}\right)=\int_{0}^{\infty} \int_{0}^{\infty} \frac{1}{\pi} \int_{0}^{\pi / 2} \exp \left[\left(\frac{\gamma_{S R} \gamma_{R D}\left|x_{q}\right|^{2}}{\gamma_{R D}+C}\right.\right. \\
& \left.\left.+\gamma_{S D}\left|x_{q}\right|^{2}\right) /\left(2 \sin ^{2} \theta\right)\right] f_{\gamma_{S D}}(\gamma) f_{\gamma_{S R D}}(\gamma) d \theta d \gamma_{S R D} d \gamma_{S D} \\
& =\frac{1}{\pi} \int_{0}^{\pi / 2} M_{\gamma_{S R D}}\left(\frac{\left|x_{q}\right|^{2}}{2 \sin ^{2} \theta}\right) M_{\gamma_{S D}}\left(\frac{\left|x_{q}\right|^{2}}{2 \sin ^{2} \theta}\right) d \theta
\end{aligned}
$$

Taking the upper bound approximation method for equation (22) yields

$$
\operatorname{PEP}\left(x_{l} \rightarrow x_{\tilde{l}}\right) \cong \frac{1}{\pi} M_{\gamma_{S R D}}\left(\frac{\left|x_{q}\right|^{2}}{2}\right) M_{\gamma_{S D}}\left(\frac{\left|x_{q}\right|^{2}}{2}\right)
$$

Substituting (17) and (14) into (23) yields

$$
\begin{array}{r}
\operatorname{PEP}\left(x_{l} \rightarrow x_{\tilde{l}}\right) \cong \frac{1}{\pi}\left[\frac{1}{\bar{\gamma}_{C} \bar{\gamma}_{R D}^{N_{r}-1}}\left(\frac{1}{\bar{\gamma}_{C}}+\frac{\left|x_{q}\right|^{2}}{2}\right)^{-N_{r}}\right. \\
\left.+\left(\frac{1}{\bar{\gamma}_{C}}-\frac{1}{\bar{\gamma}_{R D}}\right) \sum_{n=0}^{N_{r}-2} \frac{1}{\bar{\gamma}_{R D}{ }^{n}}\left(\frac{1}{\bar{\gamma}_{C}}+\frac{\left|x_{q}\right|^{2}}{2}\right)^{-(n+1)}\right] \\
\times \frac{1}{\bar{\gamma}_{S D}{ }^{N_{r}}}\left(\frac{1}{\bar{\gamma}_{S D}}+\frac{\left|x_{q}\right|^{2}}{2}\right)^{-N_{r}}
\end{array}
$$

By substituting (24) into (21), the closed-form expression of $P_{a}$ can be finally achieved. It is shown that this expression has the value very close to the simulated one.

Assuming the transmit antenna index is perfectly detected, $P_{d}$ can be derived as

$$
P_{d}=\int_{0}^{\infty} B E R(\gamma) f_{\gamma_{u p}}(\gamma) d \gamma
$$

where $B E R(\gamma)$ denotes the BER expression of $M$-QAM or $M$-PSK.

For $M$-QAM

$$
\operatorname{BER}(\gamma)=\sum_{l}^{\pi(M)} \alpha_{l} \operatorname{erfc}\left(\sqrt{\beta_{l} \gamma}\right)
$$

where $\operatorname{erfc}(\cdot)$ denotes the complementary error rate function, $\left\{\alpha_{l}, \beta_{l}, \pi(M)\right\}$ are coefficients associated with specific modulation [11].Substituting (19) and (26) into (25), the $P_{d}$ of $M$-QAM is derived as $(27)$, where $w_{C}=\beta_{l}+1 / \bar{\gamma}_{C}$, $w_{S D}=\beta_{l}+1 / \bar{\gamma}_{S D}$.
For $M$-PSK, the BER expression can be tightly approximated as [12]

$B E R(\gamma) \approx \frac{1}{\max \left(\log _{2} M, 2\right)} \sum_{k=1}^{\max (M / 4,1)} \operatorname{erfc}\left(\sqrt{\gamma} \sin \frac{(2 i-1) \pi}{M}\right)$

Substituting (19) and (28) into (25), the $P_{d}$ of $M$-PSK is derived as (29), where $\tilde{w}_{C}=\sin ^{2} \frac{(2 k-1) \pi}{M}+1 / \bar{\gamma}_{C}, \tilde{w}_{S D}=$ $\sin ^{2} \frac{(2 k-1) \pi}{M}+1 / \bar{\gamma}_{S D}$.

Equations (27) and (29) are closed-form expressions of $P_{d}$, which will be shown to agree the simulation well. Moreover, they have the values slightly smaller than the real ones because the tightly upper bound of output SNR is used.

Substituting (21), (24) and (27), (29) into (20), the overall average BERs of the system with MQAM and MPSK are, respectively, obtained, and they have the values close to the simulated ones.

\section{SIMULATION RESULTS}

In this section, we will use the derived theoretical formula to assess the performance of the cooperative AF-SM system over Rayleigh flat fading channel. In simulation, the number of transmit antenna $N_{t}=4$, and the number of receive antenna $N_{r}$ is equal to 1 or 2 . The simulation results are obtained by $10^{7}$ Monte-Carlo channel realizations, and are shown in Fig.2, Fig.3 and Fig.4.

Fig. 2 shows the error probability of the transmit antenna index estimation $\left(P_{a}\right)$ for cooperative AF-SM system. The theoretical $P_{a}$ is calculated by (21). From Fig.2, it is found that the analytical expressions are tight. This is because the $\gamma_{u p}$ in (12) takes the upper bound of true output SNR, and it results in the decreased upper bound of the error probability of transmit antenna index estimation in (21). As a result, the obtained theoretical values from (21) are very close to the true ones. Correspondingly, the theoretical $P_{a}$ is in agreement with the simulated one. As the modulation size increases, the $P_{a}$ increases accordingly. Namely, the $P_{a}$ of 16QAM is higher than that of $8 \mathrm{PSK}$. The reason for this is that higher-order modulation is easy to produce error due to the smaller Euclidean distance between the constellation points. Besides, the system with $N_{r}=2$ has lower $P_{a}$ than that with $N_{r}=1$ because of more diversity gain. The results indicate the derived $P_{a}$ is effective and reasonable. 


$$
\begin{aligned}
& P_{d, M P S K}=\sum_{k=1}^{\max (M / 4,1)} \frac{1}{\max \left(\log _{2} M, 2\right) \bar{\gamma}_{C} \bar{\gamma}_{R D}^{N_{r}-1} \bar{\gamma}_{S D}^{N_{r}}}\left\{\sum_{i=0}^{N_{r}-1} E_{N_{r}-i} \bar{\gamma}_{C}^{N_{r}-i}\left[1-\sin \frac{(2 k-1) \pi}{M} \sum_{p=0}^{N_{r}-i-1} \frac{\Gamma(p+1 / 2) \tilde{w}_{C}^{-(p+1 / 2)}}{\sqrt{\pi} \bar{\gamma}_{C}^{p} \cdot p !}\right]\right. \\
& +\sum_{j=0}^{N_{r}-1} B_{N_{r}-j} \bar{\gamma}_{S D}^{N_{r}-j}\left[1-\sin \frac{(2 k-1) \pi}{M} \sum_{q=0}^{N_{r}-j-1} \frac{\Gamma(q+1 / 2) \tilde{w}_{S D}^{-(q+1 / 2)}}{\sqrt{\pi} \bar{\gamma}_{S D}^{q} \cdot q !}\right]+\sum_{k=1}^{\max (M / 4,1)} \frac{1}{\max \left(\log _{2} M, 2\right) \bar{\gamma}_{S D}^{N_{r}}} \\
& \cdot\left(\frac{1}{\bar{\gamma}_{C}}-\frac{1}{\bar{\gamma}_{R D}}\right) \sum_{n=0}^{N_{r}-2} \frac{1}{\bar{\gamma}_{R D}{ }^{n}}\left\{\sum_{x=0}^{n} C_{n+1-x} \bar{\gamma}_{C}^{n+1-x}\left[1-\sin \frac{(2 k-1) \pi}{M} \sum_{a=0}^{n-x} \frac{\Gamma(a+1 / 2) \tilde{w}_{C}^{-(a+1 / 2)}}{\sqrt{\pi} \bar{\gamma}_{C}^{a} \cdot a !}\right]\right. \\
& \left.+\sum_{y=0}^{N_{r}-1} D_{N_{r}-y} \bar{\gamma}_{S D}^{N_{r}-y}\left[1-\sin \frac{(2 k-1) \pi}{M} \sum_{b=0}^{N_{r}-y-1} \frac{\Gamma(b+1 / 2) \tilde{w}_{S D}^{-(b+1 / 2)}}{\sqrt{\pi} \bar{\gamma}_{S D}^{b} \cdot b !}\right]\right\}
\end{aligned}
$$

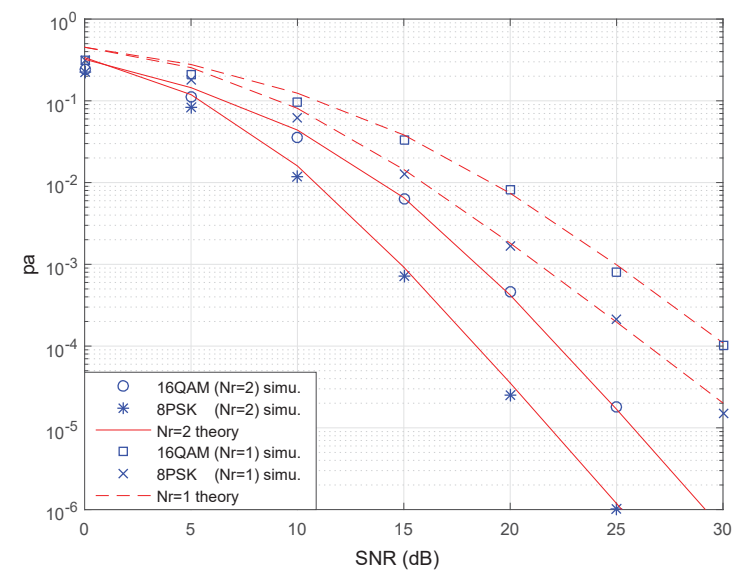

Figure 2: Error probability of antenna index estimation for AF-SM $\left(P_{a}\right)$

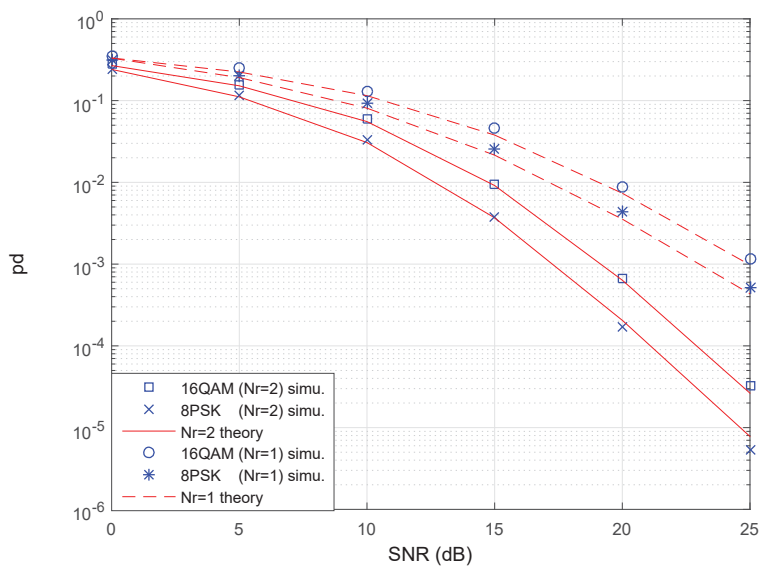

Figure 3: Error probability of symbol estimation for AF-SM $\left(P_{d}\right)$
Fig. 3 illustrates the error probability of symbol estimation $\left(P_{d}\right)$ for cooperative AF-SM system. The theoretical $P_{d}$ values of 16QAM and 8PSK are calculated by (27) and (29), respectively, and they have values very close to the simulation. Moreover, these values are slightly lower than the corresponding simulated ones. This is because the tightly upper bound of output SNR is employed. As shown in Fig.3, the $P_{d}$ of 8 PSK is lower than that of 16QAM due to the larger Euclidean distance between the constellation points. Besides, the $P_{d}$ of the system with $N_{r}=2$ is lower than that with $N_{r}=1$ because more antennas are used. The results show the derived $P_{d}$ is also effective and reasonable.

Fig. 4 plots the average BER of the cooperative AF-SM system with different receive antennas. Form Fig.4, we can see that the analytical average BER matches the simulation well because of better approximation. Moreover, the BER performance decreases as the modulation order increases. Namely, compared with the system with 16QAM, the system with 8PSK has lower BER. With the increase of the number of receiver antennas, the BER performance of the system with $N_{r}=2$ is superior to that of $N_{r}=1$ as expected due to larger diversity gain.

\section{CONCLUSIONS}

By combing spatial modulation with the AF relay-aided cooperative communication effectively, we have presented a cooperative AF-SM scheme, and investigated the performance of AF-SM in Rayleigh fading channel. Based on the performance analysis, a tightly upper bound of effective output SNR and its PDF are derived. Using the obtained PDF and the MGF, the error probability of antenna index estimation and the error probability of symbol estimation are, respectively, derived. As a result, tightly approximate closed-form expressions are obtained. With these expressions, the closedform average BER of AF-SM is achieved, and thus the system performance can be evaluated effectively in theory. Computer simulation shows that the theoretical analysis is valid and in agreement with simulation result, and the conventional need for Monte Carlo simulation is avoided. Moreover, the BER performance can be improved by increasing the receive antenna. 


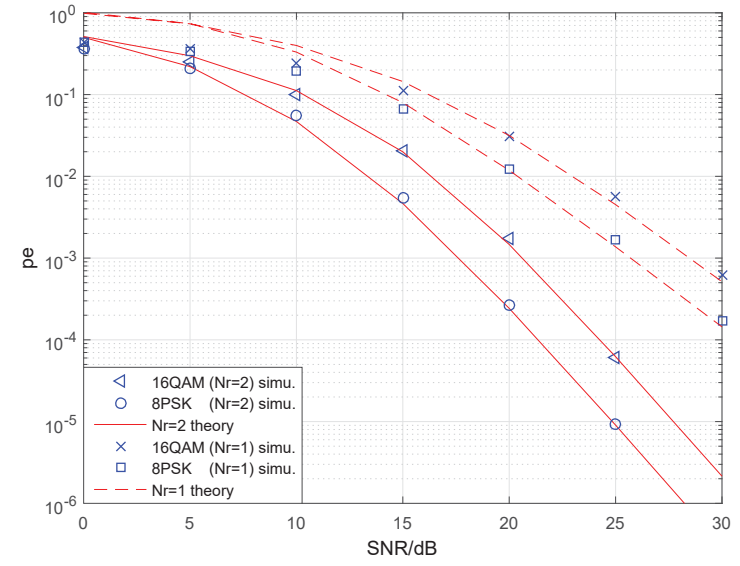

Figure 4: Average BER of AF-SM with different receive $\operatorname{antennas}\left(P_{e}\right)$

\section{ACKNOWLEDGMENT}

This work is supported by National Natural Science Foundation of China $(61571225,61571224)$, Foundation of Graduate Innovation Center in Nanjing University of Aeronautics and Astronautics (kfjj20170410), Open Research Fund of National Mobile Communications Research Laboratory of Southeast University (2017D03), Six Talent Peaks Project in Jiangsu (DZXX-007).

\section{REFERENCES}

[1] C. T. K. Ng and H. Huang. 2010. Linear precoding in cooperative MIMO cellular networks with limited coordination clusters. IEEE J. Sel. Areas Commun., 28, 9 (December 2010), 11461454.

[2] J. N. Laneman, G. W. Wornell, and D. N. C. Tse. 2001. An efficient protocol for realizing cooperative diversity in wireless networks. in Proc. IEEE ISIT'01, Washington, DC. June 2001, 294.

[3] R. Y. Mesleh, H. Haas, S. Sinanovic, C. W. Ahn, and S. Yun 2008. Spatial modulation. IEEE Trans. Veh. Technol., 57, 4 (January 2008), 2228-2241.

[4] D. Yang, C. Xu, L.-L. Yang, and L. Hanzo. 2011. Transmitdiversity-assisted space-shift keying for colocated and distributed/cooperative MIMO elements. IEEE Trans. Veh. Tech, 60, 6 (July 2011), 2864-2869.

[5] R. Mesleh, S. S. Ikki, and M. Alwakeel. 2011. Performance analysis of space shift keying with amplify and forward relaying. IEEE Commun. Lett., 15, 12 (December 2011), 1350-1352.

[6] R. Mesleh, S. S. Ikki, and M. Alwakeel. 2012. On the performance of dual-hop space shift keying with single amplify-and-forward relay. in Proc. IEEE WCNC'12, April 2012, 776-780.

[7] J. Jeganathan, A. Ghrayeb, L. Szczecinski, and A. Ceron. 2009. Space shift keying modulation for MIMO channels. IEEE Trans. Wirel. Commun., 8, 4 (July 2009), 3692-3703.

[8] P. A. Anghel and M. Kaveh. Exact symbol error probability of a cooperative network in a Rayleigh-fading environment. IEEE Trans. Wirel. Commun., 3, 5 (September 2004), 1416-1421.

[9] S. Ikki and M. H. Ahmed. 2007. Performance analysis of cooperative diversity wireless networks over Nakagami-m fading channel. IEEE Commun.Lett., 11, 4 (July 2007), 334-336.

[10] A. Papoulis. 1991. Probability, random variables, and stochastic process, McGraw-Hill.

[11] k. Cho and D. Yoon. 2002. On the general BER expression of one-and-two-dimensional amplitude modulations. IEEE Trans. Commun., 50, 7 (July 2002), 1074-1080.
[12] J. H. Lu, K. B. Letaief, J. C. Chuang and M. L. Liou. 1999. MPSK and M-QAM BER computation using signal-space concepts. IEEE Trans. Commun., 47, 2 (February 1999), 181 - 184, 